\title{
Beauty at High $\{$ Precision Sensitivity
}

Chris Quigg

Fermilab

This manuscript has been authored by Fermi Research Alliance, LLC under Contract No. DE-AC02-07CH11359 with the U.S. Department of Energy, Office of Science, Office of High Energy Physics.

$$
\text { Beauty } 2019 \text { - Ljubljana · September 30, } 2019
$$


Origin Story ...

CERN COURIER

EPS Budapest 1977

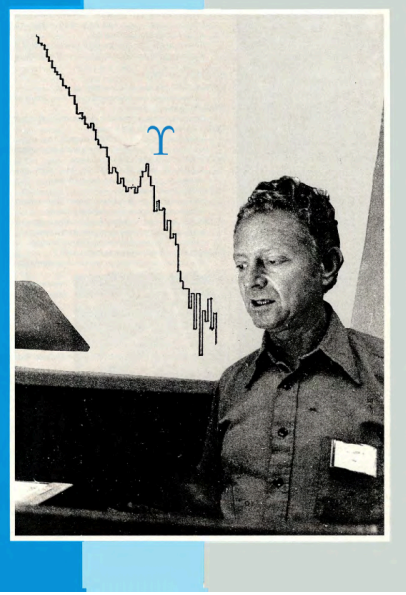

Chris Quigg
400-GeV pN $\rightarrow \mu^{+} \mu^{-}+X$

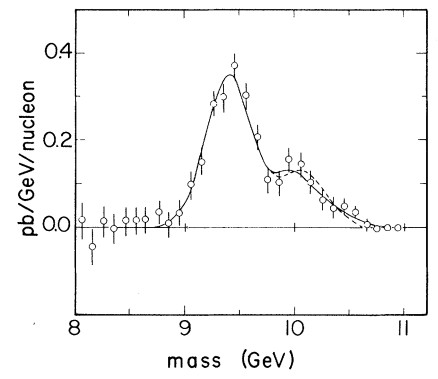

\begin{tabular}{|ccc|}
\hline E288 & $M\left(\Upsilon^{\prime}\right)-M(\Upsilon)$ & $M\left(v^{\prime \prime}\right)-M\left(\Upsilon^{\prime}\right)$ \\
\hline Two-level fit & $650 \pm 30 \mathrm{MeV}$ & \\
Three-level fit & $610 \pm 40 \mathrm{MeV}$ & $1000 \pm 120 \mathrm{MeV}$ \\
$M\left(\psi^{\prime}\right)-M(\mathrm{~J} / \psi)$ & $\approx 590 \mathrm{MeV}$ & \\
\hline
\end{tabular}

General motivation: $J / \psi, \tau$ discoveries Kobayashi-Maskawa CPV insight 
Eichten \& Gottfried: CESR Proposal (November 1976)

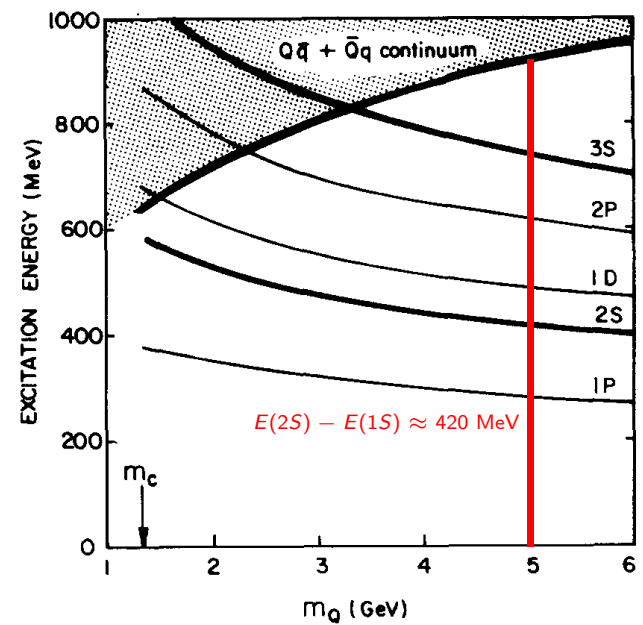

General: \# of narrow ${ }^{3} S_{1}$ levels $\propto \sqrt{M_{Q}}$ 


\section{Why choose $M_{Q}=5 \mathrm{GeV}$ ?}

Excess events at high inelasticity observed in $\bar{\nu}_{\mu} N \rightarrow \mu^{+}+$anything

$$
V-A: d \sigma(\nu q) / d y \propto 1 \quad d \sigma(\bar{\nu} q) / d y \propto(1-y)^{2}
$$

"high-y anomaly" could be explained by

$$
\left(\begin{array}{l}
u \\
b
\end{array}\right)_{\mathrm{R}} \text { with } m_{b} \approx 4-5 \mathrm{GeV}
$$

Also at Budapest $1977 \ldots$

CDHS experiment ruled out the high-y anomaly 
$\Upsilon(1 S), \Upsilon(2 S)$ leptonic widths $\leadsto Q_{b}=-\frac{1}{3}($ DORIS, 1978)

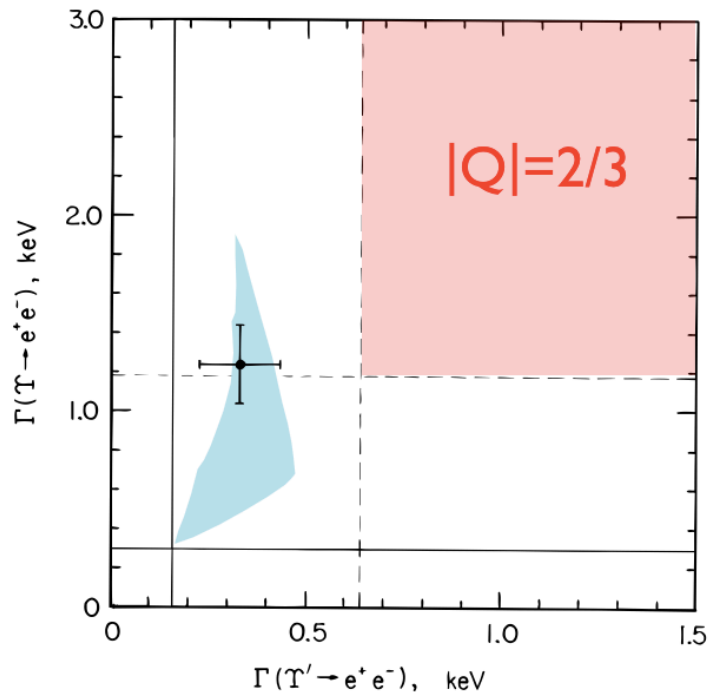


CESR resolves three narrow $\Upsilon$ states (1979-80)
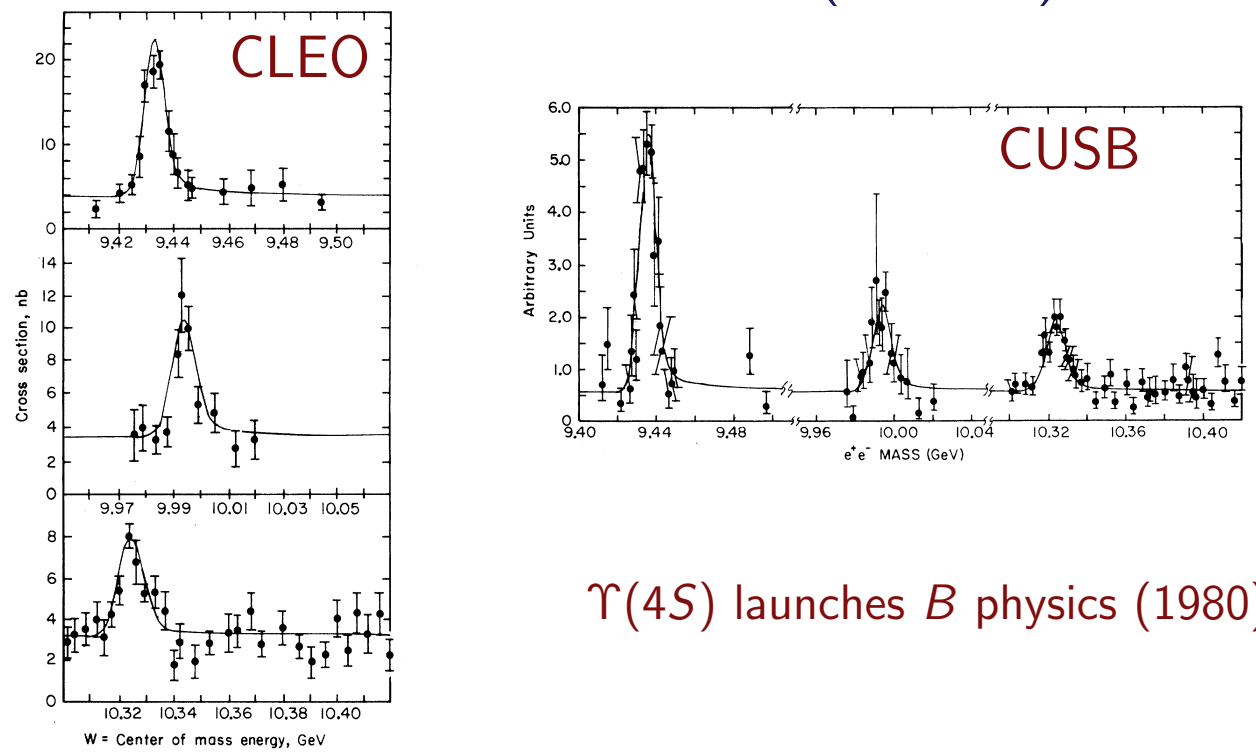

$\Upsilon(4 S)$ launches $B$ physics (1980) 


\section{Rich spectrum of $(b \bar{b})$ levels}

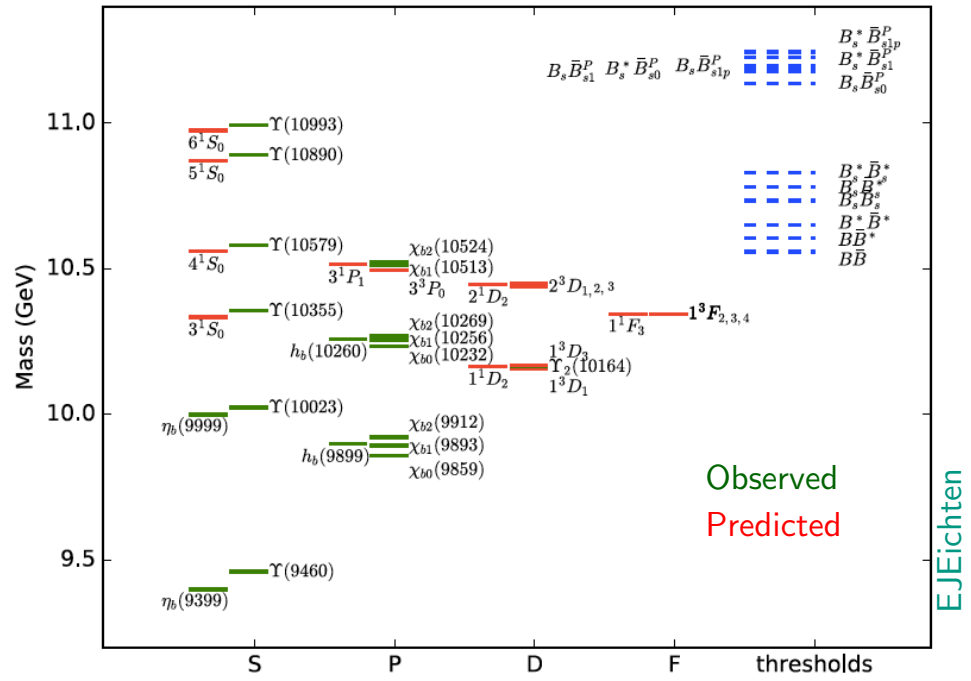

14 states below threshold still unobserved 


\section{Charmonium-associated states not pure charmonium}

All these states near or above threshold

near threshold states have possible molecule component

" $i \ldots$ ?. need more info if $J^{P C}=0^{++}, ¿ X(3915)$ ? possible $2^{3} P_{2}$

¿ $\psi(4660)$ ? possible $5 S$

$\psi(4230)$, ¿ $\psi(4360)$ ? possible hybrids

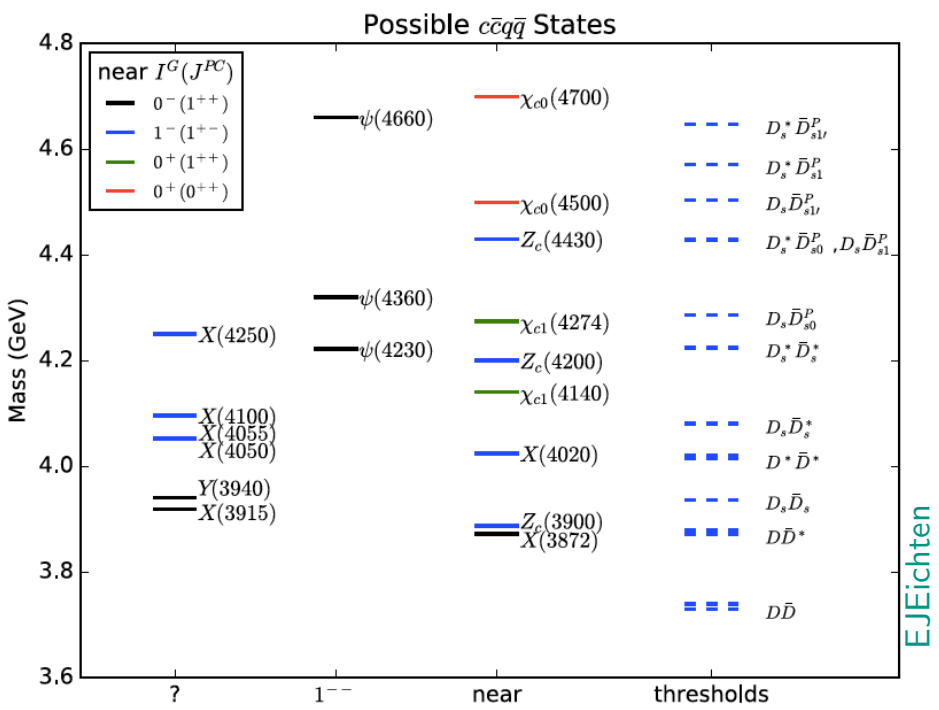

When can we find $(b \bar{b})$ analogues? 
Quarkonium-associated states: $M \gtrsim$ threshold: $X(3872)$ etc.

Mostly narrow, seen in hadronic transitions or decays

What are they?

Quarkonium (+ coupled-channels, thresholds)

Threshold effects

New body plans:

quarkonium hybrids $(q \bar{q} g)$

two-quark-two-antiquark states, including

dimeson "molecules"

tetraquarks

diquarkonium · hadroquarkonium

and superpositions!

(crypto)pentaquarks 


\section{CP violation might be large and observable (1980-81)}

\section{$C P$ Nonconservation in Cascade Decays of $B$ Mesons}

Ashton B. Carter and A. I. Sanda

Rochefeller Untuersity, New York, Nese York I0021

(Received 2 June 1980)

General techniques are introduced to expose new $C P$-nonconserving effects in cascade decays of $B$ mesons. These effects are computed in the Kobayashi-Maskawa model. The $\boldsymbol{C P}$ asymmetries so obtained range from $2 \%$ to $20 \%$ if the parameters are in the favorable range $s_{3}<s_{2} \leqslant 0.1$. Effects of this size should be observable in upcoming experiments.

\section{NOTES ON THE OBSERVABILITY OF $C P$ VIOLATIONS IN B DECAYS}

I.I. BIGI

Institut für Theor. Physik der RWTH Aachen, D-5100 Aachen, FR Germany

$$
\text { A.I. SANDA }{ }^{1}
$$

Rockefeller University, New York 10021, USA

Received 16 June 1981

We describe a general method of exposing $C P$ violations in on-shell transitions of B mesons. Such $C P$ asymmetries can reach values of the order of up to $10 \%$ within the Kobayashi-Maskawa model for plausible values of the model parameters. Our discussion focuses on those (mainly non-leptonic) decay modes which carry the promise of exhibiting clean and relatively large $C P$ asymmetries at the expense of a reduction in counting rates. Accordingly we address the complexities encountered when performing $C P$ tests with a high statistics $\mathrm{B}$ meson factory like the $\mathrm{Z}^{0}$ (and a toponium) resonance. 
Reconstruction of $B$ Mesons (CLEO, 1983)

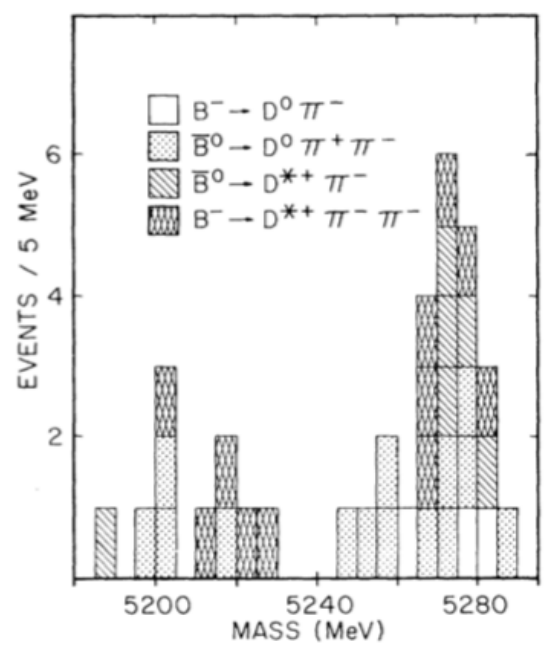

PDG: $I, J, P$ still need confirmation! 


\section{MAC \& Mark II find unexpectedly long $b$-hadron lifetime (1983)}

Charm lifetimes [fs]

$$
\begin{gathered}
D^{+}: 1040 \pm 7 \\
D^{0}: 410.1 \pm 1.5 \\
D_{s}: 504 \pm 4 \\
\Lambda_{c}: 200 \pm 6 \\
\Xi_{c}^{+}: 442 \pm 26 \\
\Xi_{c}: 112_{-10}^{+13} \\
\Omega_{c}: 268_{-26}^{+10}
\end{gathered}
$$

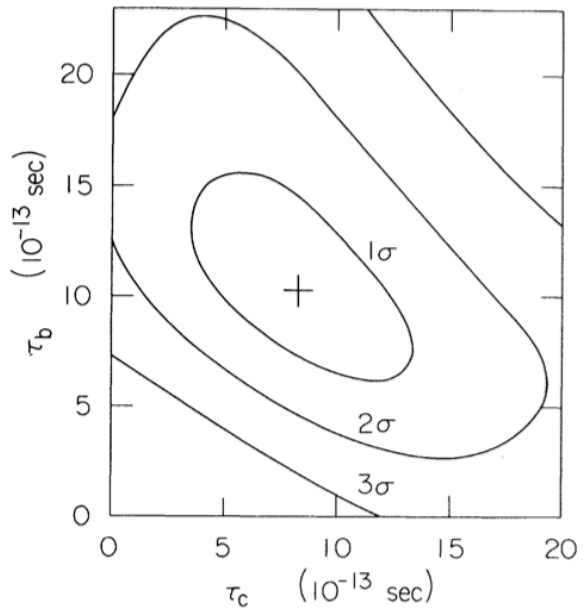

$$
\begin{gathered}
\text { Beauty lifetimes [fs] } \\
B^{+}: 1638 \pm 4 \\
B^{0}: 1519 \pm 4 \\
B_{s}: 1510 \pm 4 \\
\Lambda_{b}: 1471 \pm 9 \\
\Xi_{b}^{-}: 1572 \pm 40 \\
\Xi_{b}^{0}: 1480 \pm 30 \\
\Omega_{b}: 1640_{-170}^{+180}
\end{gathered}
$$

Evidence for small $\left|V_{c b}\right| \approx 0.05$ 


\section{$B^{0}-\bar{B}^{0}$ Mixing: the golden event from ARGUS (1987)}

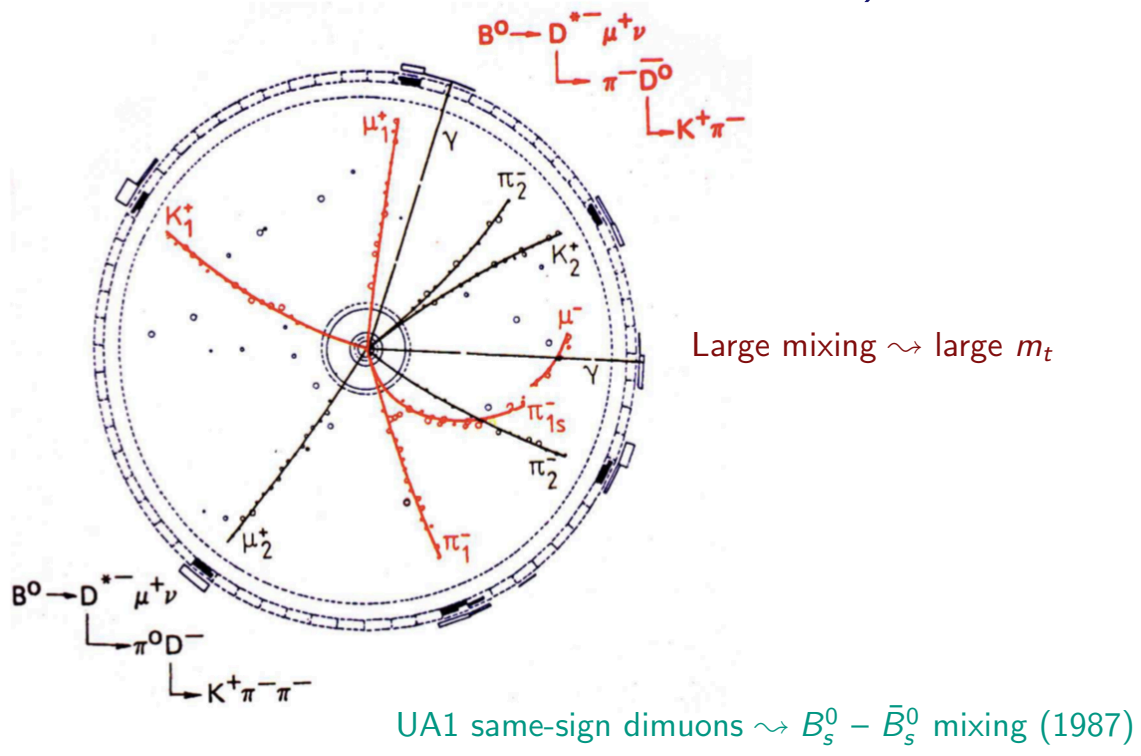


b properties imply top-quark partner must exist (1992)

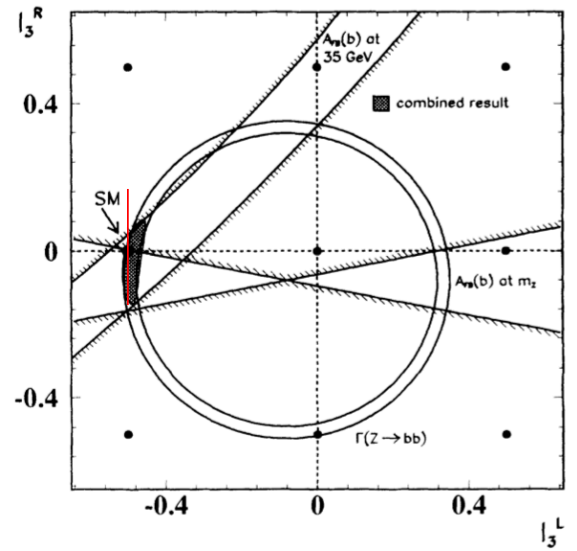

$$
L_{b} \equiv 2 I_{3 \mathrm{~L}}-2 Q_{\underline{b}} \sin ^{2} \theta_{\mathrm{W}}, R_{b} \equiv 2 I_{3 \mathrm{R}}-2 Q_{b} \sin ^{2} \theta_{\mathrm{W}}
$$

$\Gamma\left(Z^{0} \rightarrow b \bar{b}\right)$ measures $\left(L_{b}^{2}+R_{b}^{2}\right), A_{\text {peak }}^{(b \bar{b})}\left(L_{b}^{2}-R_{b}^{2}\right) /\left(L_{b}^{2}+R_{b}^{2}\right)$, LE FB asym $A(b \bar{b}) \propto\left(R_{b}-L_{b}\right)$

$$
I_{3 \mathrm{~L}}=-\frac{1}{2} ; I_{3 \mathrm{R}}=0
$$


Observation of large CP violation in $B^{0}$ decays (BABAR \& Belle, 2001)

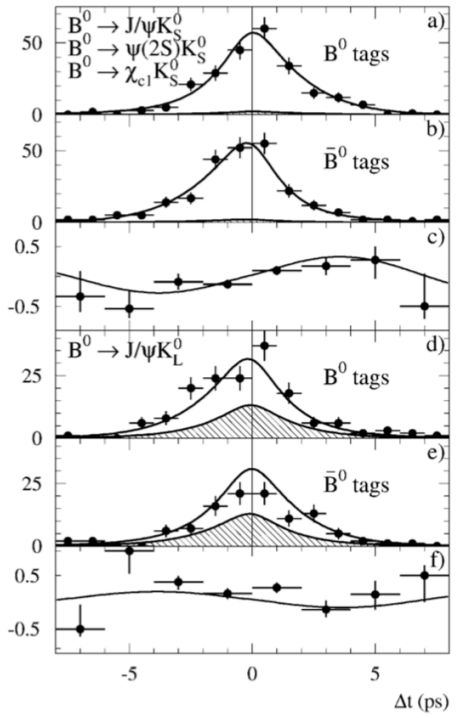

$\sin 2 \beta \approx 0.59$

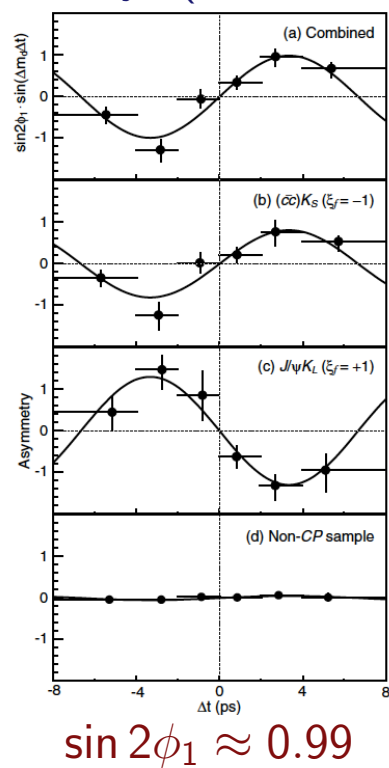




\section{Observation of $B_{s}^{0}-\bar{B}_{s}^{0}$ Oscillations (CDF, 2006)}

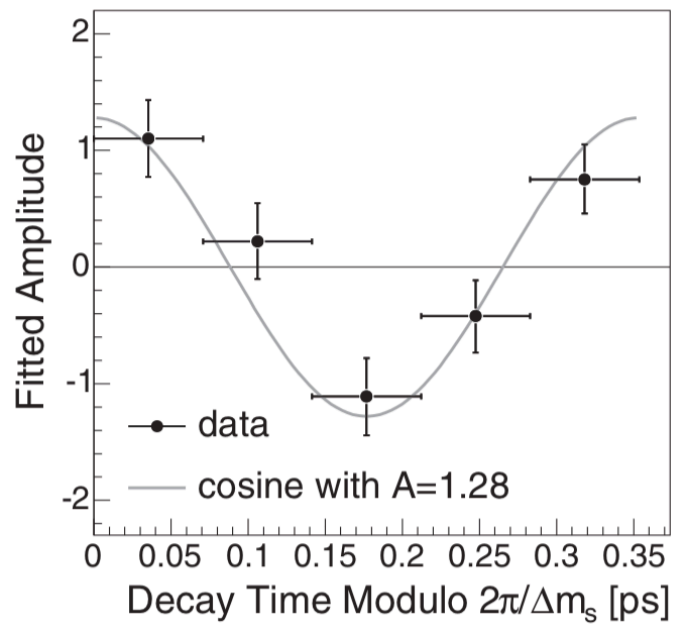

$$
\Delta m_{s} \approx 17.77 \mathrm{ps}^{-1}
$$




\section{Precision tests of the CKM paradigm}
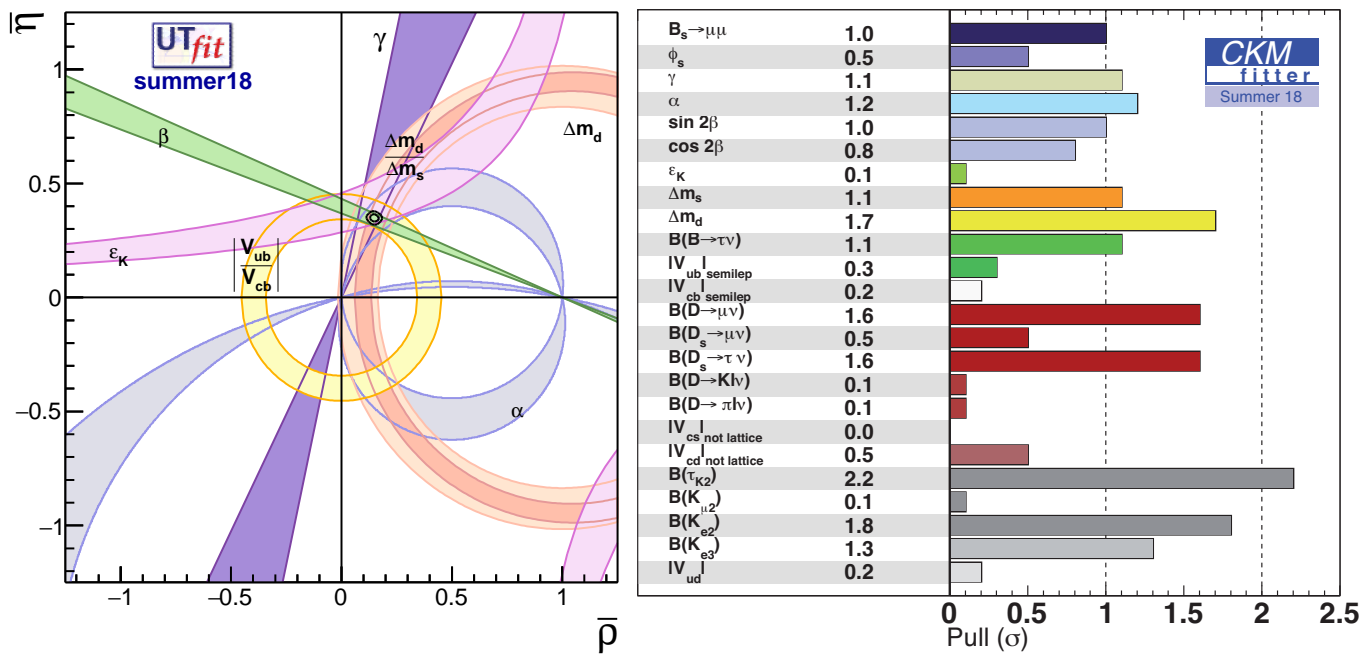
Reconstruction of $B_{c}$ meson (CDF, 2006)

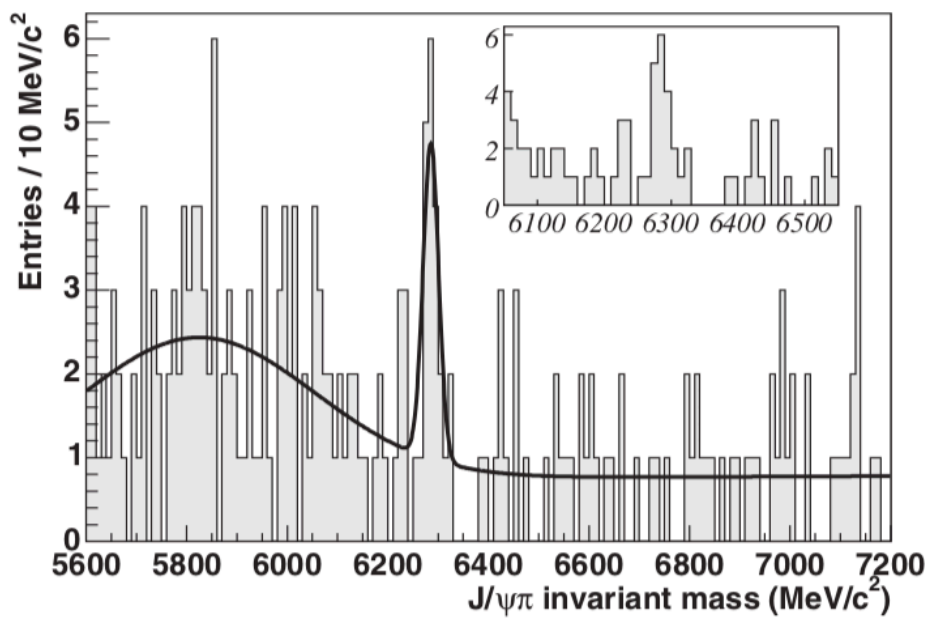

$M\left(B_{c}\right)=6274.9 \pm 0.8 \mathrm{MeV}$ (Test of lattice QCD prediction) 
Mesons with beauty and charm: stress test for NRQM, LQCD

$B_{c}$ : weak decays only

$b \rightarrow c \quad c \rightarrow s \quad b \bar{c} \rightarrow W^{-}$

$B_{c} \rightarrow J / \psi \pi:(Q \bar{Q})$ transmutation

Rich $(b \bar{c})$ excitation spectrum; interpolates $J / \psi, \Upsilon$ ( $\neq$ masses $)$

Excited states below $B D \rightarrow B_{c}+\ldots$ $B_{c}(2 S) \rightarrow B_{c}(1 S)+\pi \pi$

$P$ states: $\gamma$ transitions

Many states observable at LHC, TeraZ

Update: Eichten \& CQ (2019)

using "frozen- $\alpha_{\mathrm{s}}$ " potential, new

approach to spin splittings

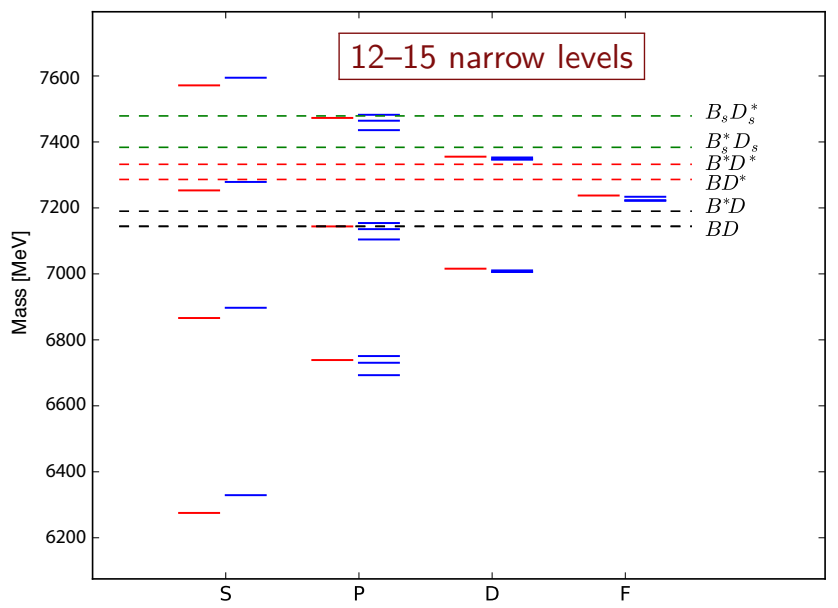




\section{Observing the $B_{c}$ spectrum: $\pi \pi$ transitions}

Combine predicted production rates (BCVEGPY2.2) with calculated branching fractions to obtain expectations for $\pi \pi$ transition rates $\leadsto$ peak heights: $B_{c}^{* \prime} / B_{c}^{\prime} \approx 2.5$

$\mathrm{M} 1 B_{c}^{*} \rightarrow \not / B_{c}$ unobserved

$\left[M\left(B_{c}^{* \prime}\right)-M\left(B_{c}^{\prime}\right)\right]-\left[M\left(B_{c}^{*}\right)-M\left(B_{c}\right)\right]$ $\approx-23 \mathrm{MeV}$ : $B_{c}^{* \prime}$ lower peak

$2 S \rightarrow \pi \pi+1 S$ transitions observed by ATLAS, CMS, LHCb

CMS separation: $-29 \mathrm{MeV}$
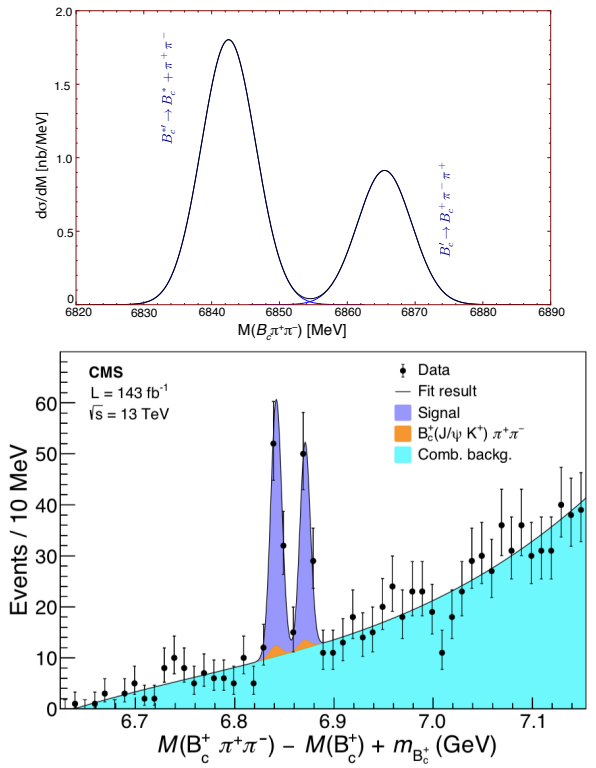


\section{Observing the $B_{c}$ spectrum: E1 transitions}

E1 spectroscopy in the $(b \bar{b})$ family: LHC experiments discovered $\chi_{b 1}^{\prime \prime}, \chi_{b 2}^{\prime \prime}$.

Incentive for the search: $2 S \rightarrow 2 P$ and $2 P \rightarrow 1 S$ transitions, assuming missing $B_{c}^{*} \rightarrow B_{c} \not /$ in the reconstruction.

$3 S, 3 P$ yields $\approx \frac{1}{4} \times 2 P \rightarrow 1 S$ lines, but higher $\gamma$ energies may aid detection. $3^{3} P_{2}(7154) \rightarrow B_{c}^{*} \gamma(777 \mathrm{MeV})$

Encourage search for $(3,2) P(b \bar{c})$.
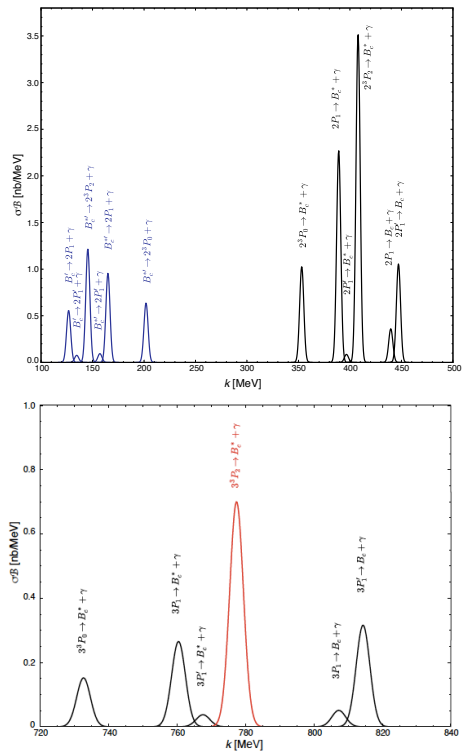
Mesons with beauty and charm: states above flavor threshold $3 S$ states above threshold have significant decay widths
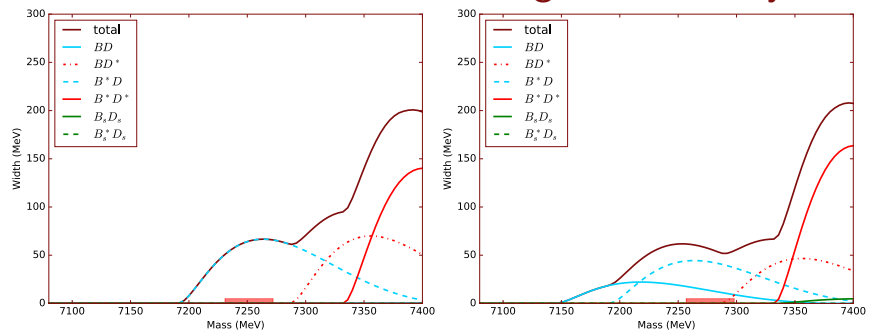

$3 P$ states just below threshold; $J=1$ may have significant mixing

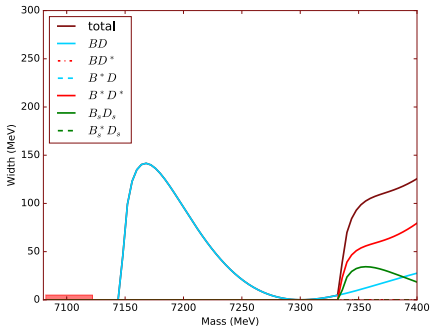

Chris Quigg

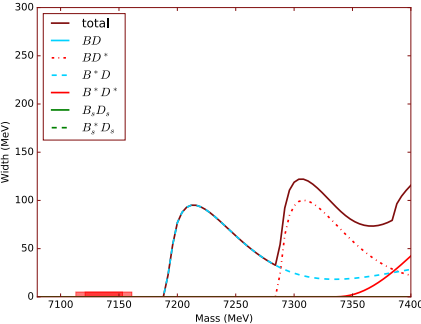

Beauty 2019 Opening

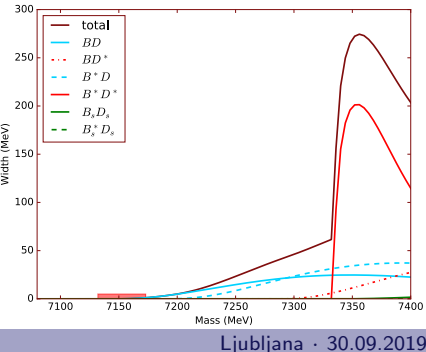




\section{Strong dynamics greatly simplifies for $M_{Q} \gg \Lambda_{Q C D}$}

Symmetry independent of dynamics of light degrees of freedom

Heavy-light systems: $(c \bar{q}),(b \bar{q}),(c q q),(b q q),(c c q),(c b q),(b b q)(q=u, d, s)$ HQET: systematic expansion in powers of $\Lambda_{\mathrm{QCD}} / M_{Q}$ HQS relations among spectra in $[(c \bar{q}),(b \bar{q}),(c c q),(b c q),(b b q)]$ and $[(c q q),(b q q)]$ QED analogue: hydrogen atom $\left(e^{-} p^{+}\right)$

Nonrelativistic $(Q \bar{Q})$ : bound-state masses $\mathcal{M} \approx 2 M_{Q}$

NRQCD: systematic expansion in powers of $v / c$

Quarkonium systems: $(c \bar{c}),(b \bar{b}),(b \bar{c})$

heavy quark velocity: $p_{Q} / M_{Q} \approx v / c \ll 1$

binding energy: $2 M_{Q}-\mathcal{M} \approx M_{Q} v^{2} / c^{2}$

QED analogs: positronium $\left(e^{+} e^{-}\right)$, "true" muonium $\left(\mu^{+} \mu^{-}\right)$, muonium $\left(\mu^{+} e^{-}\right)$ 
Heavy quark symmetry $\Rightarrow$ stable heavy tetraquarks $Q_{i} Q_{j} \bar{q}_{k} \bar{q}_{l}$
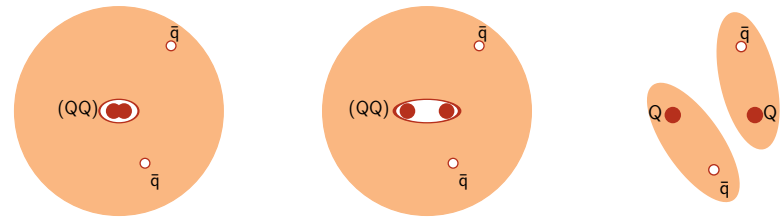

$H Q S$ relates $D H T Q$ mass to masses of $Q Q q, Q q q, Q \bar{q}$.

Lightest $b b \bar{u} \bar{d}, b b \bar{u} \bar{s}, b b \bar{d} \bar{s}$ states: (likely) no strong decays.

Heavier $b b \bar{q}_{k} \bar{q}_{l}, c c \bar{q}_{k} \bar{q}_{l}, b c \bar{q}_{k} \bar{q}_{l} \rightarrow Q \bar{q}+Q \bar{q}$ might be seen

as "double-flavor" resonances near threshold.

Observing a weakly decaying double-beauty state would establish the existence of tetraquarks and illuminate the role of

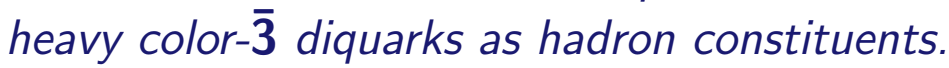


HQS relations for ground-state tetraquark masses

$$
m\left(Q_{i} Q_{j} \bar{q}_{k} \bar{q}_{l}\right)-m\left(Q_{i} Q_{j} q_{m}\right)=m\left(Q_{x} q_{k} q_{l}\right)-m\left(Q_{x} \bar{q}_{m}\right)
$$

+ finite-mass corrections

RHS is determined from data

One doubly heavy baryon observed, $\Xi_{c c}$; others from model calculations ${ }^{\star}$

$$
\text { LHCb: } M\left(\Xi_{c c}^{++}\right)=3621.40 \pm 0.78 \mathrm{MeV}
$$

*We adopt Karliner \& Rosner, PRD 90, 094007 (2014)

Strong decays $\left(Q_{i} Q_{j} \bar{q}_{k} \bar{q}_{l}\right) \not \rightarrow\left(Q_{i} Q_{j} q_{m}\right)+\left(\bar{q}_{k} \bar{q}_{l} \bar{q}_{m}\right) \forall$ ground states

Consider decays to pairs of heavy-light mesons case-by-case 
Expectations for ground-state tetraquark masses, in $\mathrm{MeV}$

\begin{tabular}{lcccc} 
State & $J^{P}$ & $m\left(Q_{i} Q_{j} \bar{q}_{k} \bar{q}_{l}\right)$ & Decay Channel & $\mathcal{Q}[\mathrm{MeV}]$ \\
\hline$\{c c\}[\bar{u} \bar{d}]$ & $1^{+}$ & 3978 & $D^{+} D^{* 0} 3876$ & 102 \\
$\{c c\}\left[\bar{q}_{k} \bar{s}\right]$ & $1^{+}$ & 4156 & $D^{+} D_{s}^{*+} 3977$ & 179 \\
$\{c c\}\left\{\bar{q}_{k} \bar{q}_{l}\right\}$ & $0^{+}, 1^{+}, 2^{+}$ & $4146,4167,4210$ & $D^{+} D^{0}, D^{+} D^{* 0} 3734,3876$ & $412,292,476$ \\
{$[b c][\bar{u} \bar{d}]$} & $0^{+}$ & 7229 & $B^{-} D^{+} / B^{0} D^{0} 7146$ & 83 \\
{$[b c]\left[\bar{q}_{k} \bar{s}\right]$} & $0^{+}$ & 7406 & $B_{s} D 7236$ & 170 \\
{$[b c]\left\{\bar{q}_{k} \bar{q}_{l}\right\}$} & $1^{+}$ & 7439 & $B^{*} D / B D^{*} 7190 / 7290$ & 249 \\
$\{b c\}[\bar{u} \bar{d}]$ & $1^{+}$ & 7272 & $B^{*} D / B D^{*} 7190 / 7290$ & 82 \\
$\{b c\}\left[\bar{q}_{k} \bar{s}\right]$ & $1^{+}$ & 7445 & $B_{s}^{*} 7282$ & 163 \\
$\{b c\}\left\{\bar{q}_{k} \bar{q}_{l}\right\}$ & $0^{+}, 1^{+}, 2^{+}$ & $7461,7472,7493$ & $B D / B^{*} D 7146 / 7190$ & $317,282,349$ \\
$\{b b\}[\bar{u} \bar{d}]$ & $1^{+}$ & 10482 & $B^{-} \bar{B}^{* 0} 10603$ & -121 \\
$\{b b\}\left[\bar{q}_{k} \bar{s}\right]$ & $1^{+}$ & 10643 & $\bar{B}_{s}^{*} / \bar{B}_{s} \bar{B}^{*} 10695 / 10691$ & $-\mathbf{4 8}$ \\
$\{b b\}\left\{\bar{q}_{k} \bar{q}_{l}\right\}$ & $0^{+}, 1^{+}, 2^{+}$ & $10674,10681,10695$ & $B^{-} B^{0}, B^{-} B^{* 0} 10559,10603$ & $115,78,136$ \\
\hline
\end{tabular}

Cf. M. Karliner \& J. L. Rosner model, Phys. Rev. Lett. 119, 202001 (2017) [arXiv:1707.07666]. Estimate deeper binding, so additional $b c$ and $c c$ candidates. 
Real-world candidates for stable tetraquarks

$J^{P}=1^{+}\{b b\}[\bar{u} \bar{d}]$ meson, bound by $121 \mathrm{MeV}$

(77 MeV below $\left.B^{-} \bar{B}^{0} \gamma\right)$

$$
\mathcal{T}_{[\bar{u} \bar{u}]}^{\{b b\}}(10482)^{-} \rightarrow \Xi_{b c}^{0} \bar{p}, B^{-} D^{+} \pi^{-} \text {, and } \underbrace{B^{-} D^{+} \ell^{-} \bar{\nu}}_{\text {manifestly weak! }}
$$

$J^{P}=1^{+}\{b b\}[\bar{u} \bar{s}]$ and $\{b b\}[\bar{d} \bar{s}]$ mesons, bound by $48 \mathrm{MeV}$

(3 $\mathrm{MeV}$ below $B B_{s} \gamma$ )

$$
\mathcal{T}_{[\bar{u} \bar{s}]}^{\{b b\}}(10643)^{-} \rightarrow \Xi_{b c}^{0} \bar{\Sigma}^{-} \quad \mathcal{T}_{[\bar{d} \bar{s}]}^{\{b b\}}(10643)^{0} \rightarrow \Xi_{b c}^{0}\left(\bar{\Lambda}, \bar{\Sigma}^{0}\right)
$$




\section{Unstable doubly heavy tetraquarks}

Resonances in "wrong-sign" (double flavor) combinations $D D, D B, B B$ ?

$J^{P}=1^{+} \mathcal{T}_{[\bar{d} \bar{s}]}^{\{c\}++}(4156) \rightarrow D^{+} D_{s}^{*+}:$ prima facie evidence for non-q $\bar{q}$ level Double charge / double charm

(New kind of resonance: no attractive force at the meson-meson level.)

$$
\begin{array}{ccc}
\text { Also, } 1^{+} \mathcal{T}_{\{\bar{q} k \bar{q}\}\}}^{\{b b\}}(10681)^{0,-,--}, \mathcal{Q}=+78 \mathrm{MeV} \quad 1^{+} \mathcal{T}_{[\bar{u} \bar{c}]}^{\{b c\}}(7272)^{0}, \mathcal{Q}=+82 \mathrm{MeV} \\
0^{+} \mathcal{T}_{[\bar{u} \bar{d}]}^{[b c]}(7229)^{0}, \mathcal{Q}=+83 \mathrm{MeV} \quad 1^{+} \mathcal{T}_{[\bar{u} \bar{d}]}^{\{c c\}}(3978)^{+}, \mathcal{Q}=+102 \mathrm{MeV}
\end{array}
$$

Aside: ${ }^{3} \mathrm{D}_{3}$ and ${ }^{3} \mathrm{~F}_{4} c \bar{c}$ mesons still to be identified in $D \bar{D}$, etc.

$\mathrm{LHC} b{ }^{3} \mathrm{D}_{3}$ candidate (2019) 


\section{Homework for experiment}

$\mathcal{T} 1$. Look for double-flavor resonances near threshold.

T2. Measure cross sections for final states containing 4 heavies: $Q_{i} \bar{Q}_{i} Q_{j} \bar{Q}_{j}$.

T3. Discover and determine masses of doubly-heavy baryons. needed to implement HQS calculation of tetraquark masses intrinsic interest in these states:

compare heavy-light mesons, possible core excitations Resolve $\Xi_{c c}^{+}$uncertainty (SELEX/LHCb)

T4. Find stable tetraquarks through weak decays. Lifetime: $\sim \frac{1}{3}$ ps ?? 


\section{Homework for theory}

T5. Develop expectations for production. A. Ali et al., "Prospects of discovering stable double-heavy tetraquarks at a Tera- $Z$ factory," arXiv:1805.02535 $\rightarrow$ PLB.

T6. Refine lifetime estimates for stable states.

T7. Understand how color configurations evolve with $Q Q$ (and $\bar{q} \bar{q}$ ) masses. J.-M. Richard, et al., "Few-body quark dynamics for doubly-heavy baryons and tetraquarks," arXiv:1803.06155, Phys. Rev. C 97, 035211 (2018).

$\mathcal{T}$ 8. Investigate stability of different body plans in the heavy-quark limit.

... up to $\left(Q_{i} Q_{j}\right)\left(Q_{k} Q_{l}\right)\left(Q_{m} Q_{n}\right): B=2$, but $Q_{p} Q_{q} Q_{r}$ color structure? 
Flavor: the problem of identity

What makes an electron an electron, a top quark a top quark, ...?

We do not have a clear view of how to approach the diverse character of the constituents of matter

CKM paradigm: extraordinarily fruitful framework in hadron sector

BUT-many parameters: no clue what determines them, nor at what energy scale they are set

Even if Higgs mechanism explains how masses and mixing angles arise, we do not know why they have the values we observe Physics beyond the standard model! 


\section{Flavor: the problem of identity (continued)}

Parameters of the Standard Model

\begin{tabular}{cl}
\hline 3 & Coupling parameters, $\alpha_{\mathrm{s}}, \alpha_{\mathrm{em}}, \sin ^{2} \theta_{\mathrm{W}}$ \\
2 & Parameters of the Higgs potential \\
1 & Vacuum phase (QCD) \\
6 & Quark masses \\
3 & Quark mixing angles \\
1 & CP-violating phase \\
3 & Charged-lepton masses \\
3 & Neutrino masses \\
3 & Leptonic mixing angles \\
1 & Leptonic CP-violating phase $(+$ Majorana phases? $)$ \\
\hline $26^{+}$ & Arbitrary parameters \\
\hline
\end{tabular}


Questions concerning the problem of identity

F1. Can we find evidence of right-handed charged-current interactions? Is nature built on a fundamentally asymmetrical plan, or are the right-handed weak interactions simply too feeble for us to have observed until now, reflecting an underlying hidden symmetry?

F2. What is the relationship of left-handed and right-handed fermions?

F3. Are there additional electroweak gauge bosons, beyond $W^{ \pm}$and $Z$ ?

F4. Are there additional kinds of matter?

F5. Is charged-current universality exact?

What about lepton-flavor universality? 


\section{$B_{(s, d)} \rightarrow \ell^{+} \ell^{-}$search and observation}

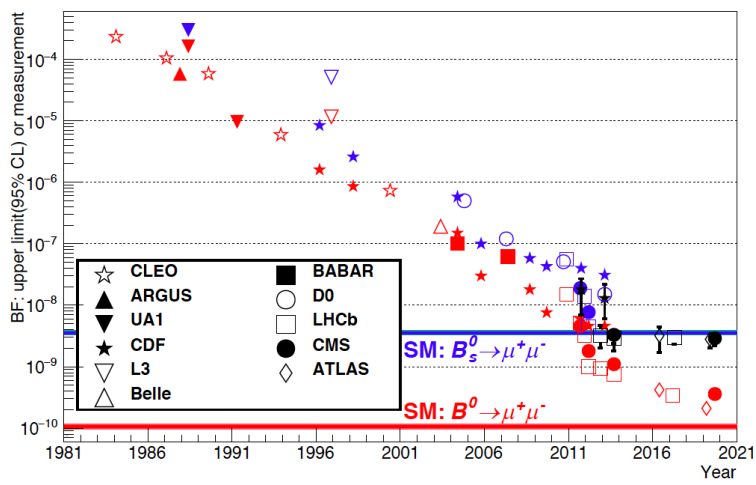

$\mathrm{SM}: \mathcal{B}\left(B_{s} \rightarrow \mu^{+} \mu^{-}\right)=(3.66 \pm 0.23) \times 10^{-9}$ $\mathcal{B}\left(B_{d} \rightarrow \mu^{+} \mu^{-}\right)=(1.06 \pm 0.09) \times 10^{-10}$
CMS Preliminary $36 \mathrm{fb}^{-1}(13 \mathrm{TeV})+20 \mathrm{fb}^{-1}(8 \mathrm{TeV})+5 \mathrm{fb}^{-1}(7 \mathrm{TeV})$

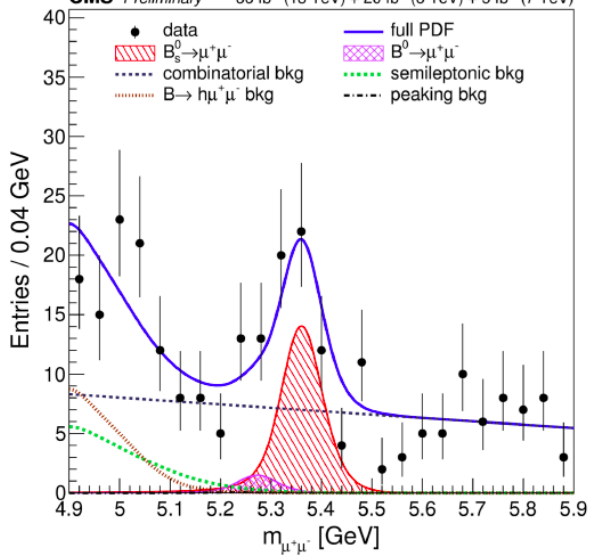

Recent CMS: $\mathcal{B}\left(B_{s} \rightarrow \mu^{+} \mu^{-}\right)=\left[2.9_{-0.4}^{+0.7} \pm 0.2\left(f_{s} / f_{d}\right)\right] \times 10^{-9}$

Coming: $\tau\left(B_{s} \rightarrow \mu^{+} \mu^{-}\right), B_{(d, s)} \rightarrow e^{+} e^{-}$searches 


\section{$K^{+} \rightarrow \pi^{+} \nu \bar{\nu}$ search and observation}
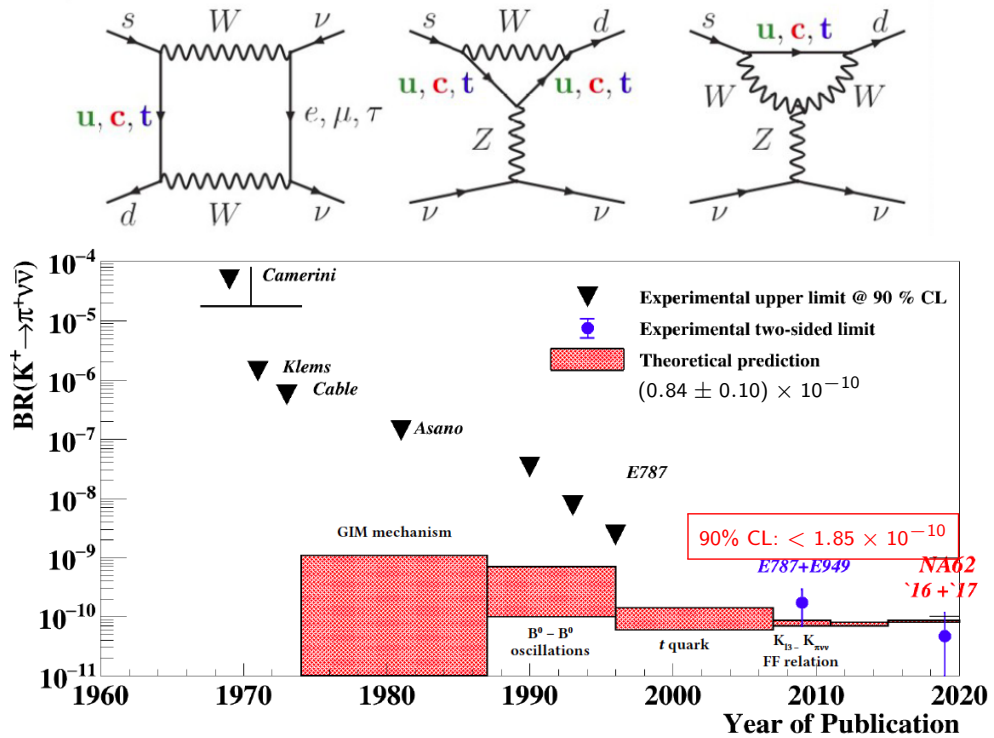
Searches for flavor-changing neutral currents

F6. Where are flavor-changing neutral currents in quark transitions? In the standard model, these are absent at tree level and highly suppressed by the Glashow-Iliopouolos-Maiani mechanism. They arise generically in proposals for physics beyond the standard model, and need to be controlled. And yet we have made no sightings! Why not?

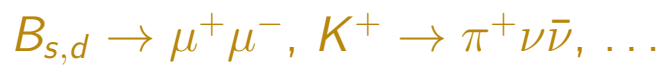

F7. Can we detect flavor-violating decays $H(125) \rightarrow \tau^{ \pm} \mu^{\mp}, \ldots$ ?

F8. How well can we test the standard-model correlation among $\mathcal{B}\left(K^{+} \rightarrow \pi^{+} \nu \bar{\nu}\right), \mathcal{B}\left(B_{s} \rightarrow \mu^{+} \mu^{-}\right)$, and the quark-mixing matrix parameter $\gamma$ ? 
Have we found the "periodic table" of elementary particles?

Pointlike spin- $1 / 2$ constituents $\left(r<10^{-18} \mathrm{~m}\right)$

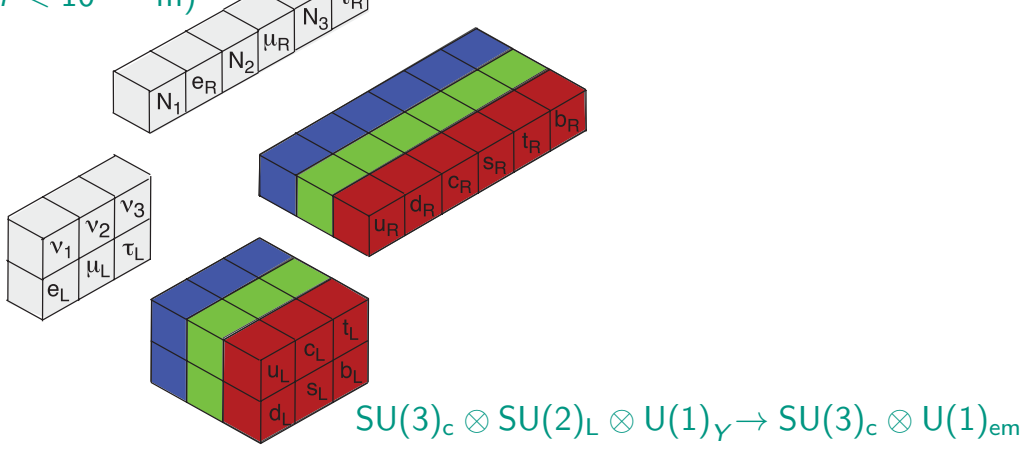

F9. What do generations mean? Is there a family symmetry?

F10. Why are there three families of quarks and leptons? (Is it so?)

F11. Are there new species of quarks and leptons?

exotic charges? 
More questions concerning the problem of identity

F12. Is there any link to a dark sector?

F13. What will resolve the disparate values of $\left|V_{u b}\right|$ and $\left|V_{c b}\right|$ measured in inclusive and exclusive decays?

F14. Is the $3 \times 3(\mathrm{CKM})$ quark-mixing matrix unitary?

F15. Why is isospin a good symmetry? What does it mean?

F16. Can we find evidence for charged-lepton flavor violation?

F17. Will we establish and diagnose a break in the SM?

F18. Do flavor parameters mean anything at all?

Contrast the landscape perspective.

F19. If flavor parameters have meaning (beyond engineering information), what is the meta-question? 
The top quark touches many topics in particle physics

t1. How well can we constrain $V_{t b}$ in single-top production, ...?

t2. How well can we constrain the top-quark lifetime? How free is $t$ ?

Recent ATLAS: $\Gamma(t)=1.9 \pm 0.5 \mathrm{GeV}(\mathrm{SM} 1.32 \mathrm{GeV})$

t3. Are there $t \bar{t}$ resonances?

t4. Can we find evidence of flavor-changing top decays $t \rightarrow(Z, \gamma)(c, u)$ ? 
Questions about EWSB and the Higgs Sector

$H 1$. Is $H(125)$ the only member of its clan? Might there be others - charged or neutral —at higher or lower masses?

H2. Does $H(125)$ fully account for electroweak symmetry breaking? Does it match standard-model branching fractions to gauge bosons? Are absolute couplings to $W$ and $Z$ as expected in the standard model?

H3. Are all production rates as expected? Any surprise sources of $H(125)$ ?

H4. What accounts for the immense range of fermion masses?

H5. Is the Higgs field the only source of fermion masses?

Are fermion couplings proportional to fermion masses? $\mu^{+} \mu^{-}$soon? How can we detect $H \rightarrow c \bar{c}$ ?

$e^{+} e^{-}$?? (basis of chemistry)

H6. What role does the Higgs field play in generating neutrino masses? 
More questions about EWSB and the Higgs Sector

H7. Can we establish or exclude decays to new particles? Does $H(125)$ act as a portal to hidden sectors? When can we measure $\Gamma_{H}$ ?

H8. Can we detect flavor-violating decays $\left(\tau^{ \pm} \mu^{\mp}, \ldots\right)$ ?

H9. Do loop-induced decays $(g g, \gamma \gamma, \gamma Z)$ occur at standard-model rates?

H10. What can we learn from rare decays $(J / \psi \gamma, \Upsilon \gamma, \ldots)$ ?

H11. Does the EW vacuum seem stable, or suggest a new physics scale?

H12. Can we find signs of new strong dynamics or (partial) compositeness?

H13. Can we establish the HHH trilinear self-coupling?

H14. How well can we test the notion that $H$ regulates Higgs-Goldstone scattering, i.e., tames the high-energy behavior of WW scattering?

H15. Is the electroweak phase transition first-order?

See Dawson, Englert, Plehn, arXiv:1808.01324 $\leadsto$ Phys. Rep. 
An exercise for all of us

How do you assess the scientific potential for Beauty and in general of
(a) The High-Luminosity LHC?
(b) The High-Energy LHC?
(c) A 100-TeV pp Collider (FCC-hh)?
(d) A 250-GeV ILC?
(e) A circular Higgs factory (FCC-ee or CEPC)?
(f) A 380-GeV CLIC?
(g) $A \mu^{+} \mu^{-} \rightarrow H$ Higgs factory?
(h) $\mathrm{LHeC} / \mathrm{FCC}$-eh? (or an electron-ion collider?)
(i) A muon-storage-ring neutrino factory?
(j) A multi-TeV muon collider?
(k) The instrument of your dreams? 